\title{
Three-Particle Complexes in Two-Dimensional Semiconductors
}

\author{
Bogdan Ganchev, ${ }^{1}$ Neil Drummond, ${ }^{1}$ Igor Aleiner, ${ }^{2,1}$ and Vladimir Fal'ko ${ }^{1}$ \\ ${ }^{1}$ Department of Physics, Lancaster University, Lancaster LA1 4YB, United Kingdom \\ ${ }^{2}$ Physics Department, Columbia University, New York, New York 10027, USA
}

(Received 18 August 2014; published 11 March 2015)

\begin{abstract}
We evaluate binding energies of trions $X^{ \pm}$, excitons bound by a donor or acceptor charge $X^{D(A)}$, and overcharged acceptors or donors in two-dimensional atomic crystals by mapping the three-body problem in two dimensions onto one particle in a three-dimensional potential treatable by a purposely developed boundary-matching-matrix method. We find that in monolayers of transition metal dichalcogenides the dissociation energy of $X^{ \pm}$is typically much larger than that of localized exciton complexes, so that trions are more resilient to heating, despite the fact that their recombination line in optics is less redshifted from the exciton line than the line of $X^{D(A)}$.
\end{abstract}

DOI: 10.1103/PhysRevLett.114.107401

Atomic layers of hexagonal transition metal dichalcogenides (TMDCs) [1-4] represent a new class of systems whose optical properties attract a lot of interest [4-10] due to their promise for applications in optoelectronics. These two-dimensional (2D) crystals are believed to be direct band gap semiconductors [11-15], and their luminescence spectra contain distinct lines interpreted as the electron-hole recombination from neutral $X$ and charged excitons (trions $X^{ \pm}$) [4,16-24], which also coexist with the recombination of excitons localized at defects.

Here, we study binding energies of acceptor- or donorbound excitons $\left(X^{A(D)}\right)$, trions $\left(X^{ \pm}\right)$, and charged acceptors $\left(A^{+}\right)$or donors $\left(D^{-}\right)$in atomic $2 \mathrm{D}$ crystals using a method developed specifically to tackle such three-body problems in two dimensions. For the trions, we also employ the diffusion quantum Monte Carlo approach $[25,26]$. We take into account a specific feature of atomically thin crystals of TMDCs, where, due to the polarizability of atomic orbitals, the interaction between charges $q_{i, j}$ is logarithmic, $\left(q_{i} q_{j} / r_{*}\right) \ln \left(r_{i j} / r_{*}\right)$, up to a distance $r_{*}$ much larger than the excitonic Bohr radius [27], as indicated by the comparison of measured [42] and calculated [42-44] spectra of ground and excited states of free excitons.

In Fig. 1 we display the calculated binding energies $\tilde{\epsilon}$ of all charged three-particle complexes, which determine the activation energy needed to dissociate them into a neutral complex and a free carrier $\left(X^{ \pm} \rightarrow X+e / h\right.$; $X^{D(A)} \rightarrow D^{0}\left(A^{0}\right)+h / e$ ). For the parametric range $0.5<$ $\left(\mu_{e} / \mu_{h}\right)<2$, which covers the cases of $\mathrm{MoS}_{2}, \mathrm{WS}_{2}$, $\mathrm{MoSe}_{2}$, and $\mathrm{WSe}_{2}$ [45], we find that the dissociation of $X^{D(A)}$ into a neutral donor (acceptor) and a hole (electron) has a much smaller activation threshold than the dissociation of a trion, which suggests that in TMDC luminescence the stronger redshifted $X^{A(D)}$ line would be more sensitive to temperature than the trion line.

Since most of the results displayed in Fig. 1 were obtained using an original approach, we describe its logic
PACS numbers: 78.20.Bh, 31.15.-p, 73.20.Hb, 78.55.-m

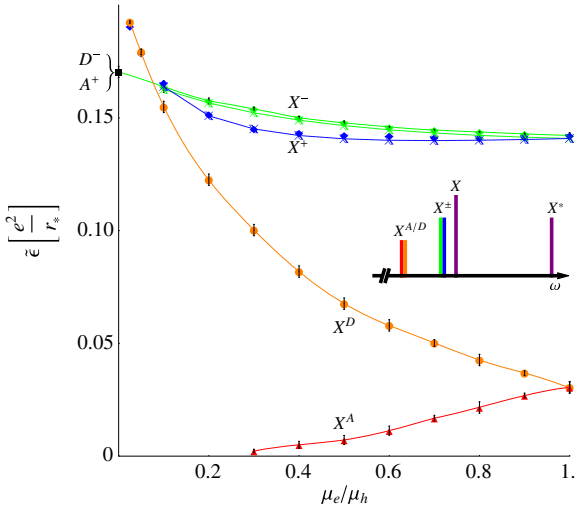

FIG. 1 (color online). Binding energies $\tilde{\epsilon}$ of charged complexes $X^{A(D)}, X^{ \pm}$, and $A^{+}$and $D^{-}$for various electron-hole mass ratios $\mu_{e} / \mu_{h}<1$ (for $\mathrm{MoS}_{2}$ and $\mathrm{MoSe}_{2}, \mu_{e} / \mu_{h} \approx 0.7$; for $\mathrm{WS}_{2}$ and $\left.\mathrm{WSe}_{2}, \mu_{e} / \mu_{h} \approx 0.6[11,15,46-49]\right)$. For trions, the results obtained by the newly developed method (diamonds) are compared to the binding energies determined using the diffusion Monte Carlo technique (crosses). Sketch: sequence of luminescence lines in TMDC spectra, including charged complexes as well as ground and first radiative excited states of the free exciton.

and theoretical features in detail, whereas the diffusion quantum Monte Carlo calculations [25,26] are discussed in the Supplemental Material [28]. Three 2D particles have six degrees of freedom, three of which correspond to center-of-mass motion and overall rotation. The quantum mechanics of the remaining degrees of freedom is equivalent to that of a particle moving in an effective threedimensional potential. The wave function $\Psi\left(\mathbf{r}_{1}, \mathbf{r}_{2}, \mathbf{r}_{3}\right)$ of three logarithmically interacting particles with masses $\mu_{1,2,3}$ [46-50] and charges $\left|q_{i}\right|=e, q_{1} q_{2}=e^{2}$, and $q_{3} q_{1,2}=-e^{2}$ obeys the Schrödinger equation $\left(\mathbf{r}_{i j} \equiv \mathbf{r}_{i}-\mathbf{r}_{j}\right)$

$$
\left[-\sum_{i=1}^{3} \frac{\hbar^{2} \nabla_{\mathbf{r}_{i}}^{2}}{2 \mu_{i}}+\frac{e^{2}}{r_{*}} \ln \frac{\left|\mathbf{r}_{31}\right|\left|\mathbf{r}_{32}\right|}{r_{*}\left|\mathbf{r}_{12}\right|}\right] \Psi=E \Psi .
$$


TABLE I. Parameters in Eqs. (1)-(4) for charged complexes in 2D semiconductors with effective electron(hole) masses $\mu_{e(h)}$.

\begin{tabular}{|c|c|c|c|c|c|c|}
\hline & \multicolumn{2}{|c|}{ Exciton $(X)$ localized on charged impurity } & \multicolumn{2}{|c|}{ Two particles localized on charged impurity } & \multicolumn{2}{|c|}{ Trion } \\
\hline & Acceptor $\left(X^{A}\right)$ & Donor $\left(X^{D}\right)$ & Acceptor $\left(A^{+}\right)$ & Donor $\left(D^{-}\right)$ & Negative trion $\left(X^{-}\right)$ & Positive trion $\left(X^{+}\right)$ \\
\hline$\underline{\mu_{1}}$ & $\mu_{e}$ & $\mu_{h}$ & $\mu_{h}$ & $\mu_{e}$ & $\mu_{e}$ & $\mu_{h}$ \\
\hline$\mu_{2}$ & $\infty$ & $\infty$ & $\mu_{h}$ & $\mu_{e}$ & $\mu_{e}$ & $\mu_{h}$ \\
\hline$\underline{\mu_{3}}$ & $\mu_{h}$ & $\mu_{e}$ & $\infty$ & $\infty$ & $\mu_{h}$ & $\mu_{e}$ \\
\hline$\underline{\theta_{1}}$ & $2 \arctan \sqrt{\mu_{e} / \mu_{h}}$ & $2 \arctan \sqrt{\mu_{h} / \mu_{e}}$ & $\pi / 2$ & $\pi / 2$ & $2 \arctan \sqrt{2 \mu_{e}+\mu_{h} / \mu_{h}}$ & $2 \arctan \sqrt{2 \mu_{h}+\mu_{e} / \mu_{e}}$ \\
\hline$\overline{\theta_{2}}$ & $\pi$ & $\pi$ & $\pi / 2$ & $\pi / 2$ & $2 \arctan \sqrt{2 \mu_{e}+\mu_{h} / \mu_{h}}$ & $2 \arctan \sqrt{2 \mu_{h}+\mu_{e} / \mu_{e}}$ \\
\hline
\end{tabular}

After separating the motion of the center of mass, $\mathbf{R}_{\mathrm{cm}}=\left(\sum_{i} \mu_{i} \mathbf{r}_{i}\right) / M, M \equiv \sum_{i} \mu_{i}$, and introducing dimensionless $\tilde{\mathbf{r}}=\mathbf{r}_{12} / r_{0}, \mathbf{r}^{\prime}=\left(\sum_{i=1}^{2} \mu_{i} \mathbf{r}_{i 3}\right) /\left(r_{0}\left[M \mu_{1} \mu_{2} / \mu_{3}\right]^{1 / 2}\right)$, where $r_{0}^{-1}=\left\{2 e^{2} \mu_{1} \mu_{2} /\left[r_{*} \hbar^{2}\left(\mu_{1}+\mu_{2}\right)\right]\right\}^{1 / 2}$, and spherical coordinates

$$
\left[\begin{array}{l}
{\left[r_{x}^{\prime}, r_{y}^{\prime}\right]} \\
{\left[\tilde{r}_{x}, \tilde{r}_{y}\right]}
\end{array}\right]=r\left[\begin{array}{c}
\cos \frac{\theta}{2}\left[\cos \left(\Phi+\frac{\phi}{2}\right), \sin \left(\Phi+\frac{\phi}{2}\right)\right] \\
\sin \frac{\theta}{2}\left[\cos \left(\Phi-\frac{\phi}{2}\right), \sin \left(\Phi-\frac{\phi}{2}\right)\right]
\end{array}\right],
$$

Eq. (1) takes the form

$$
E=\frac{\mathbf{P}_{\mathrm{cm}}^{2}}{2 M}+\frac{e^{2}}{r_{*}}\left[\frac{1}{2} \ln \frac{\hbar^{2} \prod_{i=1,2}\left(\mu_{i}+\mu_{3}\right)}{2 e^{2} r_{*} \mu_{3}^{2}\left(\mu_{1}+\mu_{2}\right)}+\epsilon\right],
$$

where $\epsilon$ are the eigenvalues of the Schrödinger equation

$$
\begin{aligned}
& {\left[-\nabla_{4}^{2}+\ln r+U(\theta, \phi)\right] \psi=\epsilon \psi,} \\
& -\nabla_{4}^{2}=-\frac{\partial^{2}}{\partial r^{2}}-\frac{3 \partial}{r \partial r}+\frac{4 \hat{\mathbf{L}}^{2}}{r^{2}}+\frac{\hat{\Theta}}{r^{2} \sin ^{2} \theta}, \\
& U(\theta, \phi)=\frac{1}{2} \ln \left[\frac{\left(1-\mathbf{n} \cdot \mathbf{n}_{1}\right)\left(1-\mathbf{n} \cdot \mathbf{n}_{2}\right)}{\left(1-\mathbf{n} \cdot \mathbf{n}_{z}\right)}\right], \\
& \mathbf{n}=[\sin \theta \cos \phi, \sin \theta \sin \phi, \cos \theta] .
\end{aligned}
$$

This transforms the three-body problem to a onebody problem in a higher-dimensional space, where $\mathbf{n}$ is a position of a fictitious particle on a sphere, $\mathbf{L}$ is a three-dimensional angular momentum operator, $\hat{\mathbf{L}}^{2}=$ $-(1 / \sin \theta)(\partial / \partial \theta) \sin \theta(\partial / \partial \theta)-\left(1 / \sin ^{2} \theta\right)\left(\partial^{2} / \partial \phi^{2}\right)$, and $\hat{\Theta}=[-(\partial / \partial \Phi)+4 \cos \theta(\partial / \partial \phi)](\partial / \partial \Phi)$. Vectors $\mathbf{n}_{z}$ and $\mathbf{n}_{i=1,2}$ characterize the direction of the maximal repulsion and attraction, respectively,

$$
\begin{aligned}
\mathbf{n}_{z} & =[0,0,1], \quad \mathbf{n}_{i}=\left[(-1)^{i} \sin \theta_{i}, 0, \cos \theta_{i}\right], \\
\tan \left(\theta_{1,2} / 2\right) & =\left[M \mu_{1,2} /\left(\mu_{3} \mu_{2,1}\right)\right]^{1 / 2},
\end{aligned}
$$

where parameters for particular complexes are specified in Table I. The color-scale visualization of $U$ is shown in the inset to Fig. 2. Classically, the particle collapses to either $\mathbf{n}_{1}$ or $\mathbf{n}_{2}$; this observation is useful for finding the large-distance asymptotic states.

Because of rotational symmetry, the potential $U$ in Eq. (3) does not depend on the angle $\Phi$. Hence, the eigenstates can be classified by the integer angular momentum $J: \Psi_{J}(r, \theta, \phi, \Phi)=e^{i J \Phi} \psi_{J}(r, \theta, \phi)$, with $J=0$ for the ground state of the three-particle complex (see the Supplemental Material [28]). For $\Psi_{0}$ to be single valued, we must have $\psi_{0}(r, \theta+2 \pi, \phi)=\psi_{0}(r, \theta, \phi+2 \pi)=$ $\psi_{0}(r,-\theta, \phi+\pi)=\psi_{0}(r, \theta, \phi)$. In general, the potential $U$ also has a mirror reflection symmetry $U(\phi)=U(-\phi)$. When two particles in the complex are identical $\left(\theta_{1}=\theta_{2}\right)$, $U(\theta)=U(-\theta)$, states are either symmetric $(s)$ or antisymmetric $(a)$ in $\theta$.

In the following, we use the conventional [54] basis of spherical harmonics $Y_{l \geq 0, m}(\theta, \phi),|m| \leq l$,

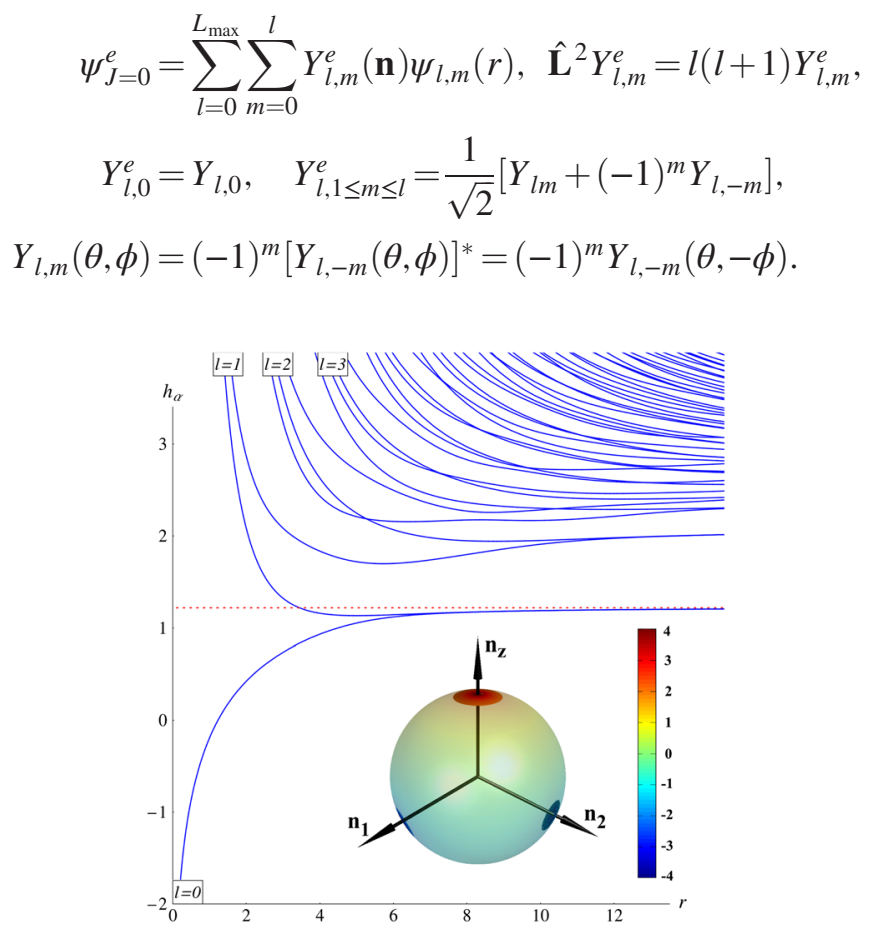

FIG. 2 (color online). Eigenvalues $h_{\alpha}(r)$ of $\hat{H}(r)$ in Eq. (6) for $\theta_{1}=\theta_{2}=(\pi / 2)$ and $L_{\max }=30$ [53]. For $r \ll 1, h_{\alpha}$ are bunched by the angular momenta $l$, whereas for $r \gg 1, a$ and $s$ doublets correspond to the particle localization in the minima $\mathbf{n}_{1,2}$ with vanishing tunneling (note that $a-s$ crossings are allowed). The red dashed line marks the boundary $X_{0}^{0}$ of the continuum spectrum for the exciton and a free particle, and at $r \gg 1$, $h(r) \approx X_{0}^{0}-c / r^{2}$, determined by the $2 \mathrm{D}$ van der Waals attraction between the charged particle and the neutral exciton, which produces an infinite number of shallow bound states. Inset: color scale image of the potential $U$ in Eq. (3). 
In this basis, Eq. (3) becomes

$$
\left[\frac{d^{2}}{d r^{2}}+3 \frac{d}{r d r}\right] \psi=(\hat{H}(r)-\epsilon \mathbb{1}) \psi
$$

where vector $\psi$ is comprised of the components $\psi_{l, m}$, and the matrix $\hat{H}(r)$ has elements

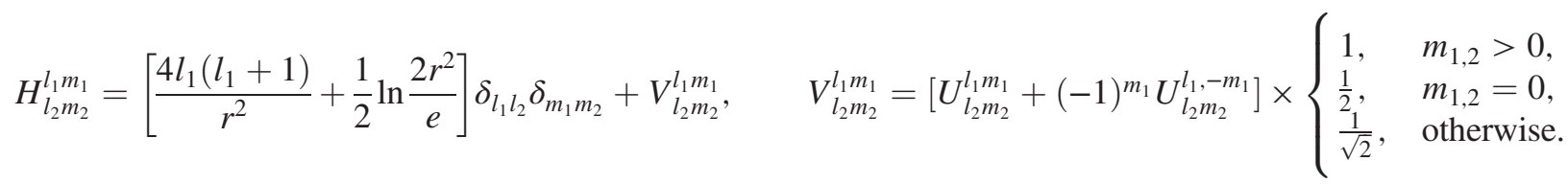

Remarkably, the matrix elements $U_{l_{2} m_{2}}^{l_{1} m_{1}}$ can be found in a closed analytic form (for the derivation, see the Supplemental Material [28])

$$
U_{l_{2} m_{2}}^{l_{1} m_{1}}=(-1)^{m_{1}} \sqrt{\pi\left(2 l_{1}+1\right)\left(2 l_{2}+1\right)} \sum_{l=l_{\min }}^{l_{1}+l_{2}} \frac{\sqrt{2 l+1}}{l(l+1)}\left(\begin{array}{ccc}
l_{1} & l & l_{2} \\
-m_{1} & m & m_{2}
\end{array}\right)\left(\begin{array}{ccc}
l_{1} & l & l_{2} \\
0 & 0 & 0
\end{array}\right)\left[Y_{l, m}^{*}\left(\mathbf{n}_{z}\right)-Y_{l, m}^{*}\left(\mathbf{n}_{1}\right)-Y_{l, m}^{*}\left(\mathbf{n}_{2}\right)\right],
$$

where $m \equiv m_{1}-m_{2}, l_{\min } \equiv \max \left(1,\left|l_{1}-l_{2}\right|,|m|\right)$, the $3 j$ symbols follow Ref. [54], and $\mathbf{n}_{i}$ are from Eq. (4).

Numerically found eigenvalues of Hamiltonian (6) are shown in Fig. 2. At $r \gg 1$, the eigenfunctions are peaked near $\mathbf{n}=\mathbf{n}_{1,2}$ suggesting an adiabatic solution for Eq. (5) at $r \gg 1$. Consider the equation

$$
\left[-\frac{1}{\rho} \frac{d}{d \rho} \rho \frac{d}{d \rho}+\frac{m^{2}}{\rho^{2}}+\ln \rho\right] \varphi_{n}^{m}(\rho)=\chi_{n}^{m} \varphi_{n}^{m}(\rho)
$$

which determines the spectrum of a $2 \mathrm{D}$ exciton with the logarithmic interaction $e^{2} / r_{*} \ln \left(\rho / r_{*}\right)$ :

$$
X_{n}^{m}=\frac{e^{2}}{r^{*}}\left[\frac{1}{2} \ln \frac{\hbar^{2}\left(\mu_{1}+\mu_{3}\right)}{2 e^{2} r_{*} \mu_{1} \mu_{3}}+\chi_{n}^{m}\right] .
$$

Integer $m$ and $n \geq 0$ are the 2D angular momentum and radial quantum number, respectively, and the interlevel distances $[42,44]$ determined by the eigenvalues listed in Table II do not depend on the masses.

The adiabatic wave function (closely bound electronhole pair and the third particle far from the pair) is

$$
\psi^{(1,2)}(r, \tilde{\theta})=\varphi_{0}^{0}(r|\sin (\tilde{\theta} / 2)|) \mathcal{F}_{\epsilon}^{1,2}(r \cos (\tilde{\theta} / 2)),
$$

where "local" coordinates near $\mathbf{n}_{1 / 2}$ on the unit sphere are introduced as $\mathbf{n}(\theta, \phi)=\cos \tilde{\theta} \mathbf{n}_{i}+\sin \tilde{\theta} \cos \tilde{\phi} \mathbf{n}_{i}^{\prime}+\sin \tilde{\theta} \sin \tilde{\phi} \mathbf{n}_{i}^{\prime \prime}$, where $\mathbf{n}_{i}^{\prime}$ and $\mathbf{n}_{i}^{\prime \prime}$ are two unit vectors orthogonal to each other

TABLE II. The eigenvalues of Eq. (7) that determine the spectrum of ground and excited states of the exciton, Eq. (8).

\begin{tabular}{llcc}
\hline \hline$\chi_{n}^{m}$ & $m=0$ & $m=1$ & $m=2$ \\
\hline$n=0$ & 0.5265 & 1.386 & 1.844 \\
$n=1$ & 1.661 & 2.009 & \\
$n=2$ & 2.177 & & \\
\hline \hline
\end{tabular}

and to $\mathbf{n}_{i}$. Representation (9a) is valid if the tunneling between the two minima is weak. Substituting Eq. (9a) into Eq. (5), treating the singular logarithmic potential exactly and the remainder in second-order perturbation theory, we find

$$
\begin{aligned}
& {\left[\frac{1}{x} \frac{d}{d x} x \frac{d}{d x}+\frac{\gamma_{1,2}^{2}}{x^{2}}\right] \mathcal{F}_{\epsilon}^{(1,2)}(x)=\tilde{\epsilon}^{(1,2)} \mathcal{F}_{\epsilon}^{(1,2)}(x),} \\
& -\tilde{\epsilon}^{(1,2)} \equiv \epsilon-\chi_{0}^{0}-\frac{1}{2} \ln \frac{2 \sin ^{2} \frac{\theta_{1}+\theta_{2}}{2}}{\sin ^{2} \frac{\theta_{1,2}}{2}},
\end{aligned}
$$

where $\tilde{\epsilon}$ is the binding energy of a complex and the dimensionless strength of the van der Waals attraction is

$$
\begin{aligned}
\gamma_{1,2}^{2} & =1.23\left[\cot \left(\theta_{1,2} / 2\right)-\cot \left(\left(\theta_{1}+\theta_{2}\right) / 2\right)\right]^{2} \\
& \equiv 1.23 \mu_{2,1}\left(\mu_{1,2}+\mu_{3}\right)^{2} /\left[M \mu_{1,2} \mu_{3}\right] .
\end{aligned}
$$

The solution corresponding to the bound state is

$$
\mathcal{F}^{(1,2)}=K_{i \gamma_{1,2}}\left(x \sqrt{\tilde{\epsilon}^{(1,2)}}\right)
$$

where $K_{i \gamma}(y)$ is the MacDonald function, and $\tilde{\epsilon}^{(1,2)}$ is determined by matching Eq. (9d) with the solution of Eq. (5)

$$
\psi_{l, m}(r) \propto r^{2 l}, \quad r \ll 1 .
$$

In the interaction region, $r \simeq 1$, the problem can only be handled numerically. Numerical solution of Eq. (5) is not practical as many states in the interaction region are evanescent (see Fig. 2), and the search for the bound state would require the finding of $N=\left(L_{\max }+1\right)\left(L_{\max }+2\right) / 2$ boundary conditions at $r \rightarrow 0$ with exponential accuracy. Instead, we employ a procedure that does not suffer from exponential dependence on $r$.

We notice that one can replace solving Eq. (5) for all $r$ with the solution on only $r>R$, where $R>0$ is an arbitrary distance, if one knows the $N \times N$ boundary condition matrix $\hat{\Lambda}$ defining the behavior of $\psi(r \rightarrow R+0)$ 


$$
[r d \psi / d r+\hat{\Lambda}(R) \psi]_{r=R}=0 .
$$

Requiring the invariance of solutions of Eq. (5) with respect to changes in $R$, we find

$$
R d \hat{\Lambda} / d R=R^{2}[\epsilon \mathbb{1}-\hat{H}(R)]-2 \hat{\Lambda}+\hat{\Lambda}^{2},
$$

where matrix multiplication is defined in the basis (5) as $[\hat{A} \hat{B}]_{l_{1} m_{1}}^{l_{2} m_{2}}=\sum_{l=0}^{L_{\max }} \sum_{m=0}^{l}[\hat{A}]_{l_{1} m_{1}}^{l m}[\hat{B}]_{l m}^{l_{2 m} m_{2}}, \hat{\Lambda}=\hat{\Lambda}^{*}=\hat{\Lambda}^{\dagger}$, and the initial condition follows from Eq. (9e)

$$
[\hat{\Lambda}(0)]_{l m}^{l^{\prime} m^{\prime}}=-2 l \delta_{l l^{\prime}} \delta_{m m^{\prime}} .
$$

The asymptotic dependence of the highest eigenvalues $\lambda_{\alpha}$ of matrix $\hat{\Lambda}(R)$ corresponds to the asymptotic wave function in Eqs. (9a) and (9d), so that for an energy $\epsilon$ corresponding to a bound state [55]

$$
\lambda_{\alpha}(R \gg 1)=-(x / \mathcal{F}) d \mathcal{F} /\left.d x\right|_{x=R} .
$$

We use Eq. (12) to find energies of bound states numerically. First, we match tangentially the numerically calculated dependence of the highest eigenvalue $\lambda_{0}(R)$ using Eq. (12) (as illustrated in Fig. 2), and find the distance $R^{(i)}$ and an overestimated binding energy $\tilde{\epsilon}^{(i)}$. Next, we choose a distance $R_{\max }, R^{(i)}<R_{\max }<L_{\max }$, to be used as a reference point in the rest of iterative procedure. Then, using Eqs. (11a) with $1 / R_{\epsilon}^{2}$ determined by the variable energy $\tilde{\epsilon}<\tilde{\epsilon}^{(i)}$, we evaluate $\hat{\Lambda}\left(R_{\max }\right)$, and its highest eigenvalue $\lambda_{\alpha}^{0}\left(R_{\max }\right)$, and find such energy $\tilde{\epsilon}$ that $\left[x d \mathcal{F} /\left.(\mathcal{F} d x)\right|_{x=R_{\max }}+\lambda_{\alpha}\left(R_{\max }\right)\right]^{2}$ is minimal. The outcome of such matching is exemplified in Fig. 3 showing the eigenvalues of matrix $\hat{\Lambda}(R)$ found for a trion [56].

The resulting binding energies, calculated for various cases listed in Table I and various electron-hole mass ratios [27] are shown Fig. 1, where, for comparison, we also show our results of the trion binding energies calculated using the diffision quantum Monte Carlo method. These two theoretical approaches give very close values, within the error bars determined by the limited size $L_{\max }$ of the sphericalharmonic basis. This agreement indicates that the new method offers an efficient tool to study complexes with more generic forms of electron-electron and electron-hole interaction, taking into account crossover from logarithmic to $1 / r$ dependence at the longest distances. Note that the results displayed in Fig. 1 for $\mu_{e}<\mu_{h}$ can be used for $\mu_{h}<\mu_{e}$ by swapping $\left(\mu_{e}, X^{+}, X^{A}\right) \leftrightarrow\left(\mu_{h}, X^{-}, X^{D}\right)$.

After comparing the binding energies of various threeparticle complexes, we conclude that the "third" charge is more weakly bound (has a smaller dissociation energy) in an exciton localized on a charged donor or acceptor than in a trion [57]. As a result, heating of a 2D crystal would suppress the luminescence from localized complexes much more than the luminescence of trions, because the evaporation of one of the optically active carriers from $X^{D(A)}$ would happen at a much lower temperature than the temperature required for the decomposition of $X^{ \pm}$. Such

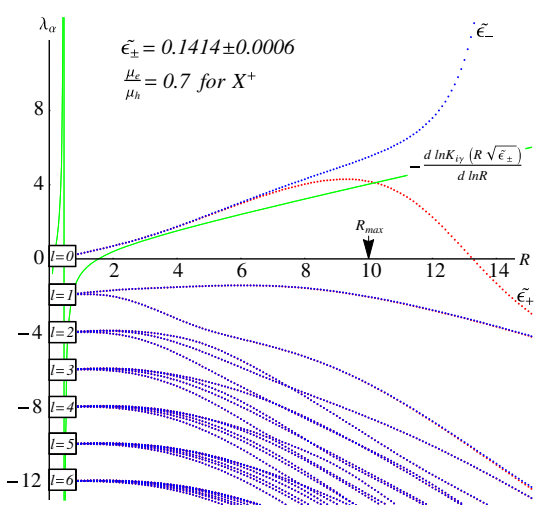

FIG. 3 (color online). Eigenvalues $\lambda_{\alpha}$ of matching matrix $\hat{\Lambda}(R)$ evaluated numerically for $\epsilon=\epsilon_{ \pm}$slightly above (blue, $\epsilon_{-}$) and slightly below (red, $\epsilon_{+}$) the bound state energy of a trion with $\mu_{e}=0.7 \mu_{h}$. The energy-sensitive highest eigenvalue is compared to the asymptotic of a logarithmic derivative in Eq. (9d) (green) calculated at the converged binding energy.

behavior is highly counterintuitive, because, despite weaker binding, the line of $X^{D(A)}$ in recombination spectra, $\omega_{X^{D(A)}}=\omega_{X}-\left(e^{2} / r_{*}\right)\left\{\tilde{\epsilon}_{X^{D(A)}}+(1 / 2) \ln \left[1+\left(\mu_{e(h)} / \mu_{h(e)}\right)\right]\right\}$, lies below (redshifted) the line of a trion, $\omega_{X^{ \pm}}=$ $\omega_{X}-\left(e^{2} / r_{*}\right) \tilde{\epsilon}_{X^{ \pm}}$. For comparable masses of electrons and holes, the exciton-trion splitting appears to be an order of magnitude smaller than the splitting between the ground state of the exciton and its first optically active excited state $X_{1}^{0}$, at $\Delta_{1}=\omega_{X^{*}}-\omega_{X}=1.14\left(e^{2} / r_{*}\right)$, whereas $\omega_{X^{D(A)}}-\omega_{X} \simeq$ $0.5 \Delta_{1}$, as prescribed by the two-particle binding energy of the electron (hole) in the donor (acceptor) being much larger than the one of the exciton, overcompensating the difference between the three-particle binding energies. Such temperature behavior of the lower end of the recombination spectra in TMDCs has recently been observed in several experiments on $\mathrm{WSe}_{2}$ [58-60].

We thank V. Cheianov, T. Heinz, and A. Morpurgo for discussions, and D. Gradinar for help in preparing the manuscript. This work was supported by EC FP7 Graphene Flagship Project No. CNECT-ICT-604391, ERC Synergy Grant No. Hetero2D, and the Simons Foundation.

[1] B. Radisavljevic, A. Radenovic, J. Brivio, V. Giacometti, and A. Kis, Nat. Nanotechnol. 6, 147 (2011).

[2] D. Lembke and A. Kis, ACS Nano 6, 10070 (2012).

[3] H. Wang, L. Yu, Y. H. Lee, Y. Shi, A. Hsu, M. Chin, L. J. Li, M. Dubey, J. Kong, and T. Palacios, Nano Lett. 12, 4674 (2012).

[4] X. Xu, W. Yao, D. Xiao, and T. F. Heinz, Nat. Phys. 10, 343 (2014).

[5] D. Xiao, G. B. Liu, W. Feng, X. Xu, and W. Yao, Phys. Rev. Lett. 108, 196802 (2012).

[6] H. Zeng, J. Dai, W. Yao, D. Xiao, and X. Cui, Nat. Nanotechnol. 7, 490 (2012).

[7] K.F. Mak, K. He, J. Shan, and T.F. Heinz, Nat. Nanotechnol. 7, 494 (2012). 
[8] G. Sallen, L. Bouet, X. Marie, G. Wang, C. R. Zhu, W. P. Han, Y. Lu, P. H. Tan, T. Amand, B.L. Liu, and B. Urbaszek, Phys. Rev. B 86, 081301 (2012).

[9] T. Cao, G. Wang, W. Han, H. Ye, C. Zhu, J. Shi, Q. Niu, P. Tan, E. Wang, B. Liu, and J. Feng, Nat. Commun. 3, 887 (2012).

[10] H. Conley, B. Wang, J. Ziegler, R. Haglund, S. Pantelides, and K. I. Bolotin, Nano Lett. 13, 3626 (2013).

[11] A. Kuc, N. Zibouche, and T. Heine, Phys. Rev. B 83, 245213 (2011).

[12] Z. Y. Zhu, Y. C. Cheng, and U. Schwingenschlögl, Phys. Rev. B 84, 153402 (2011).

[13] A. Molina-Sanchez, D. Sangalli, K. Hummer, A. Marini, and L. Wirtz, Phys. Rev. B 88, 045412 (2013).

[14] W. Jin et al., Phys. Rev. Lett. 111, 106801 (2013).

[15] A. Kormanyos, V. Zolyomi, N. D. Drummond, P. Rakyta, G. Burkard, and V. I. Falko, Phys. Rev. B 88, 045416 (2013).

[16] A. Ramasubramaniam, Phys. Rev. B 86, 115409 (2012).

[17] H. P. Komsa and A. V. Krasheninnikov, Phys. Rev. B 86, 241201 (2012).

[18] D. Y. Qiu, F. H. da Jornada, and S. G. Louie, Phys. Rev. Lett. 111, 216805 (2013)

[19] M. M. Glazov, T. Amand, X. Marie, D. Lagarde, L. Bouet, and B. Urbaszek, Phys. Rev. B 89, 201302 (2014).

[20] G. Berghauser and E. Malic, Phys. Rev. B 89, 125309 (2014).

[21] A. R. Klots, A. K. M. Newaz, B. Wang, D. Prasai, H. Krzyzanowska, D. Caudel, N. J. Ghimire, J. Yan, B. L. Ivanov, K. A. Velizhanin, A. Burger, D. G. Mandrus, N. H. Tolk, S. T. Pantelides, and K. I. Bolotin, Sci. Rep. 4, 6608 (2014).

[22] K. F. Mak, K. He, C. Lee, G. H. Lee, J. Hone, T. F. Heinz, and J. Shan, Nat. Mater. 12, 207 (2013).

[23] C. Zhang, H. Wang, W. Chan, C. Manolatou, and F. Rana, Phys. Rev. B 89, 205436 (2014).

[24] A. Srivastava, M. Sidler, A. V. Allain, D. S. Lembke, A. Kis, and A. Imamoglu, arXiv:1407.2624.

[25] D. M. Ceperley and B. J. Alder, Phys. Rev. Lett. 45, 566 (1980).

[26] R. J. Needs, M. D. Towler, N. D. Drummond, and P. López Ríos, J. Phys. Condens. Matter 22, 023201 (2010).

[27] As shown in Ref. [28], $r_{*}=a_{z}\left(\varepsilon_{\|}-1\right) / 2$, where $\varepsilon_{\|}$is the in-plane component of the dielectric permitivity tensor of the bulk layered material and $a_{z}$ is the distance between layers in it. For example, in $\mathrm{WS}_{2}$, this leads [42] to the estimate $r_{*} \sim 7.5 \mathrm{~nm}$; hence. $e^{2} / r_{*} \sim 200 \mathrm{meV}$ and a typical trion binding energy is $\sim 30 \mathrm{meV}$.

[28] See Supplemental Material at http://link.aps.org/ supplemental/10.1103/PhysRevLett.114.107401, which includes Refs. [29-41], for precise form of the particle interactions, proper coordinate transformations, matrix elements, classification of the optical transitions, and more information on Quantum Monte Carlo calculations.

[29] L. V. Keldysh, Pis'ma Zh. Eksp. Teor. Fiz. 30, 245 (1979) [JETP Lett. 30, 224 (1979)].

[30] L. S. Gradstein and I. M. Ryzhik, Tables of Integrals, Series and Products, 6th ed. (Academic Press, New York, 2000).

[31] L. D. Faddeev and S. P. Merkuriev, Quantum Scattering Theory for Several Particle Systems (Springer, New York, 1993).

[32] E. A. Hylleraas, Z. Phys. 54, 347 (1929).

[33] S. Chandrasekhar, Astrophys. J. 100, 176 (1944).

[34] M. Y. J. Tan, N. D. Drummond, and R. J. Needs, Phys. Rev. B 71, 033303 (2005).
[35] R. M. Lee, N. D. Drummond, and R. J. Needs, Phys. Rev. B 79, 125308 (2009).

[36] W. Foulkes, L. Mitas, R. Needs, and G. Rajagopal, Rev. Mod. Phys. 73, 33 (2001).

[37] N. D. Drummond, M. D. Towler, and R. J. Needs, Phys. Rev. B 70, 235119 (2004).

[38] P. López Ríos, P. Seth, N. D. Drummond, and R. J. Needs, Phys. Rev. E 86, 036703 (2012).

[39] C. J. Umrigar, K. G. Wilson, and J. W. Wilkins, Phys. Rev. Lett. 60, 1719 (1988).

[40] N. D. Drummond and R. J. Needs, Phys. Rev. B 72, 085124 (2005).

[41] C. J. Umrigar, J. Toulouse, C. Filippi, S. Sorella, and R. G. Hennig, Phys. Rev. Lett. 98, 110201 (2007).

[42] A. Chernikov, T. C. Berkelbach, H. M. Hill, A. Rigosi, Y. Li, O. B. Aslan, D. R. Reichman, M. S. Hybertsen, and T. F. Heinz, Phys. Rev. Lett. 113, 076802 (2014).

[43] I. R. Lapidus, Am. J. Phys. 49, 807 (1981).

[44] K. Eveker, D. Grow, B. Jost, C. E. Monfort, and K. W. Nelson, Am. J. Phys. 58, 1183 (1990).

[45] A. Kormanyos, G. Burkard, M. Gmitra, J. Fabian, V. Zolyomi, N. Drummond, and V. Fal'ko, arXiv:1410.6666.

[46] H. Shi, H. Pan, Y.-W. Zhang, and B. I. Yakobson, Phys. Rev. B 87, 155304 (2013).

[47] D. Wickramaratne, R. K. Lake, and F. Zahid, J. Chem. Phys. 140, 124710 (2014).

[48] N. Zibouche, P. Philipsen, T. Heine, and A. Kuc, arXiv:1403.0552.

[49] W. Zhang, Z. Huang, and W. Zhang, Nano Res. 7, 1731 (2014).

[50] Here, we neglect the interband mixing present in the $\mathbf{k} \cdot \mathbf{p}$ theory Hamiltonians developed for the description of transition metal dichalcogenides $[15,45]$, which is important for the exciton-photon coupling and Berry curvature effects in transport [51,52] but is irrelevant for the analysis of exciton and trion binding energies since those are substantially less than the band gap.

[51] M. A. Cazalilla, H. Ochoa, and F. Guinea, Phys. Rev. Lett. 113, 077201 (2014).

[52] H. Ochoa, F. Finocchiaro, F. Guinea, and V. I. Fal'ko, Phys. Rev. B 90, 235429 (2014).

[53] For several of the lowest eigenvalues, the inclusion of higher- $l$ spherical harmonics leads to a $<3 \%$ change at the distances shown.

[54] A. R. Edmonds, Angular Momentum in Quantum Mechanics, 2nd ed. (Princeton University Press, Princeton, NJ, 1960).

[55] Here, we omit the superscript $(1,2)$ as $\gamma_{1}=\gamma_{2}$ for the symmetric case. Otherwise, the strongest potential minimum has to be chosen.

[56] For a trion, $\theta_{1}=\theta_{2}$, and the iterative procedure can be used separately for symmetric $(s)$ and antisymmetric $(a)$ states.

[57] The small values of the $X^{D(A)}$ binding energies also agree with the analytical solution of Eq. (12) obtained for asymptotically shallow ground states in the potential $-\gamma^{2} / r^{2}: \tilde{\epsilon} \sim \exp \left(-\left\{\pi-2 \operatorname{Arg}\left[\Gamma\left(i \gamma_{1,2}\right)\right]\right\} / \gamma_{1,2}\right)$, where $\Gamma$ is the gamma function.

[58] C. R. Zhu, K. Zhang, M. Glazov, B. Urbaszek, T. Amand, Z. W. Ji, B. L. Liu, and X. Marie, Phys. Rev. B 90, 161302 (2014).

[59] T. Heinz (private communication).

[60] M. Potemski (private communication). 